\title{
Development of a modelling framework for optimal sequencing of water supply options at the regional scale incorporating sustainability and uncertainty
}

\author{
Eva H.Y. Beh" ${ }^{\mathrm{a}}$, Holger R. Maier ${ }^{\mathrm{a}}$ and Graeme C. Dandy \\ ${ }^{a}$ School of Civil, Environmental and Mining Engineering, The University of Adelaide, Adelaide 5005 \\ Email: ebeh@civeng.adelaide.edu.au
}

\begin{abstract}
As urban populations around the world continue to grow, achieving sustainable urban water supply is becoming crucial in order to meet increasing demand. Cyclical drought and climate change have placed further strain on water supplies and it is therefore necessary to plan to ensure the sustainability of water supply systems at a regional scale. Water supply sustainability planning (WSSP) necessitates the use of a long-term planning horizon and multiple criteria for assessment, both of which have inherent uncertainties and difficulties. The perceived inability to account for great uncertainties in long-term future demand, economic stability, climate and technological change when it comes to the supply and usage of water has resulted in planning dominated by short-term and politically motivated decision-making. In response, this paper outlines a proposed conceptual framework to optimally sequence water supply projects, incorporating sustainability and uncertainty, at the regional scale. Sequencing of water supply projects involves choosing which options to implement at which stages over a planning horizon. In the past, the sequencing of water supply projects was straightforward, as there were relatively few options (e.g. when to build the next reservoir) and the only criteria that had to be considered were water supply security and cost. However, as a result of climate variability, climate change and the increased adoption of sustainability principles, the problem of sequencing water supply projects has become much more complex. With the increase in urbanization, urban infill and diminishing natural water sources, there is an increase in the need to consider recycled water and desalinisation as additional or alternative water supply options. Therefore, where 30 years ago only reservoirs might have been considered when planning a secure water supply for a city, in the $21^{\text {st }}$ century a large number of alternative water sources are able to be considered in the sequencing approach presented in this paper. These include desalination, stormwater re-use and rainwater tanks. The proposed model framework is based on multi-objective optimisation, so that a number of competing objectives (e.g. cost, greenhouse gas emissions) can be taken into account. The aim is to find optimal mix of water supply options, and when these projects should be implemented, can be identified from among the large number of options (e.g. rainwater tanks, different stormwater schemes, desalination etc.). Long-term planning is an important aspect of sustainable water supply systems. When planning for water supply systems for the long term future in climate and demand, as well as economic and technological conditions, are highly variable and hard to predict. These factors affect the performance of water supply systems, and thus uncertainty is accounted for in the proposed model framework. The optimisation model takes the robustness of solutions into account, so that the decisions made now will be as insensitive to future changes (e.g. population growth, climate change, new technologies) as possible. Uncertainty is the key feature of most water resource system planning related to temporal variability. To ensure, therefore, that the water resource system operates in a satisfactory manner over time, continual reassessment of the system is essential in order to identify and reduce the risk of system failure. Hence, the model framework allows long term plans to be reviewed and re-optimised at regular intervals to take account of changes in external factors.
\end{abstract}

Keywords: sequencing, uncertainty, robustness, urban water supply, sustainability 
Beh et al. Development of a modeling framework for optimal sequencing of water supply options...

\section{INTRODUCTION}

The optimal sequencing of urban water supply sources has traditionally focused on the reservoir expansion problem and economic objectives (Connarty \& Dandy, 1996). However, as a result of climate variability, climate change and the increased adoption of sustainability principles, the problem of sequencing water supply projects has become much more complex, as illustrated in Figure 1.

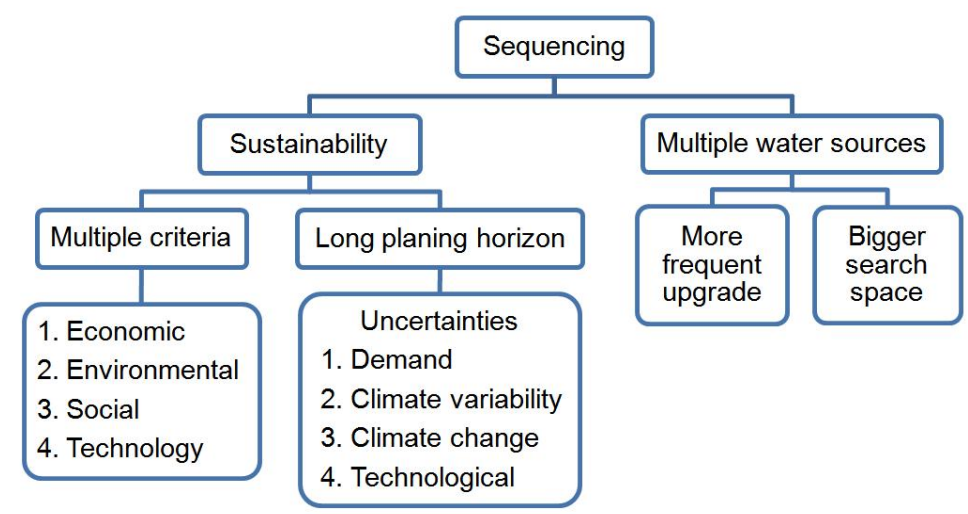

Figure 1 Criteria involved in the sequencing of sustainable water supply projects

Firstly, there has been a significant increase in the use of alternative sources of water, such as desalination, stormwater re-use and rainwater tanks in order to increase water supply security in times of drought and in response to potential climate change impacts (Coombes \& Lucas, 2006). This has resulted in a significant increase in the number of alternative water sources that need to be included in the sequencing process and increased the frequency at which water supply systems are upgraded, as many of the alternative sources of water have limited capacity. This makes is more difficult to know which combination of sources is best and when certain sources should be developed and brought online.

Secondly, the adoption of sustainability principles has resulted in the need to consider various assessment criteria in addition to the traditionally used economic criterion. Such criteria include environmental, social and technical criteria, as well as temporal scale (Hellström et al., 2000). This has increased the complexity of the sequencing task significantly, as sequences that represent optimal tradeoffs between competing objectives need to be determined, rather than just the sequence that minimises economic cost.

Thirdly, adoption of sustainability principles also requires consideration of extended time frames in the planning of water resources projects, such as 50 or 100 years (Mitchell et al., 2007). This amplifies any uncertainties that are present in the sequencing process, both on the demand and the supply side of the equation. Demand is a function of various external factors, such as population growth, household density, changes in per capita demand, industrial and commercial demand, and future land development. So, it is likely to change substantially over extended time frames (Tanaka et al., 2006). Additionally, there is a large level of uncertainty in supply due to climate change and climate variability and although progress is being made towards reducing these uncertainties, more work is required as many hydrological features occur on a smaller scale than current global circulation models (GCMs) can predict (Kundzewicz \& Somlyody, 1997).

As a result of the extended time frames, future shocks, such as changes in technology, are also likely to play a role, which could have a significant impact on the relative merits of different water sources. For example, the development of nanotube-based membranes is likely to make desalination much cheaper and less energy intensive in the future (Gethard et al., 2011). However, such shocks are even more difficult to predict than the changes in supply and demand discussed above. This further complicates the development of sequencing plans for urban water sources, particularly given the extended design life of water supply infrastructure, as once implemented, it generally lasts for a very long time, so that the impacts of decisions made now will be felt a long way into the future.

The purpose of this paper is to introduce a conceptual framework that is able to cater for the three challenges outlined above. 
Beh et al. Development of a modeling framework for optimal sequencing of water supply options...

\section{CONCEPTUAL UNDERPINNINGS OF PROPOSED FRAMEWORK}

\subsection{Introduction}

The proposed framework for dealing with the challenges outlined in Section 1 is underpinned by two main concepts: multi-objective optimization using evolutionary algorithms and the use of robustness-based objective functions to drive this optimization process. These concepts are discussed in detail in subsequent sections.

\subsection{Multi-Objective Optimisation}

In order to account for a number of competing sustainability objectives and to maximize the chances that sequences with optimal or near-optimal trade-offs between the objectives are found in the large search space of potential water supply alternatives and capacities, it is proposed that a multi-objective optimization approach based on evolutionary search strategies (e.g. genetic algorithms) should be used. Evolutionary approaches are well suited to this task, as they can be linked directly with simulation models, which are required to simulate supply and demand throughout the planning horizon, and have been found to perform well in a number of water resources applications (Paton et al., 2009).

\subsection{Robustness-Based Objective Functions}

Given the extended planning horizons associated with the consideration of sustainability principles, the uncertainty surrounding factors affecting system performance and the long design life of water supply infrastructure, it is proposed that the objective functions that are used as part of the multi-objective optimisation process should be based on the concept of robustness. Robustness is generally understood as the ability to withstand external shocks or to be stable under a range of uncertainties (Bankes, 2010). By optimizing the robustness of the sequence of water supply options, the resulting water infrastructure will be less sensitive to unknown future conditions. In other words, while the sequence of projects is not likely to be optimal for every actual condition that could occur in the future, it will perform reasonably well under a range of circumstances.

As part of the framework introduced in this paper, it is proposed to use regret as the measure of robustness to be minimized as part of the optimization process. Regret is a measure of the variation in the objective function value (e.g. cost, energy usage) of a solution (i.e. a particular sequence of water supply projects) over a range of equally likely future scenarios (e.g. climate change, population growth) and is given by:

$$
\begin{aligned}
& R_{x}=\max _{x}\left[C_{x y}-L_{x}\right] \\
& L_{x}=\min _{y}\left[C_{x y}\right]
\end{aligned}
$$

where, $\mathrm{R}_{x}$ is the maximum regret associated with sequences $x ; \mathrm{C}_{x y}$ is the total objective function value of sequences $x$ for scenarios $y ; \mathrm{L}_{y}$ is the least objective function value sequence for scenarios $y$. However, as suggested by Cui and Kuczera (2010), regret should not be considered as the sole objective, as certain solutions with low regret (e.g. low variation in cost over the scenarios considered) might have high average objective function values (e.g. high average cost over the scenarios considered), which is undesirable, assuming that the aim is to minimise the objective function. In this case, a solution with a lower average value of the objective function and a slightly higher regret might be more desirable. In order to find the most robust sequencing solution, therefore, two values should be included in the objective function of the optimisation process (i.e. average value and maximum regret) for each of the sustainability objectives (e.g. cost, greenhouse gas emissions) over the range of scenarios considered.

However, this makes it difficult to select the "best" solution, as a number of different sustainability objectives are usually considered. In order to assist with the process of finding the most appropriate solution, a number of graphical approaches can be used, including inspection of plots of regret versus average values for each sustainability objective (), which allows comparison of more objective function values for each of the solutions. 
Beh et al. Development of a modeling framework for optimal sequencing of water supply options...

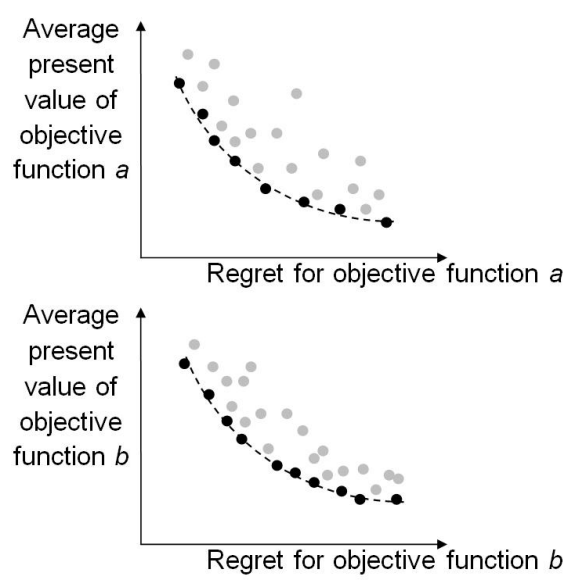

Figure 2 Expected trade-off between average objective function value and regret for each sustainability objective ), which enable consideration of a large number of solutions, and spider plots showing the trade-offs between the multiple sustainability objectives for the average and maximum regret values (Error! Reference source not found.), which allows comparison of more objective function values for each of the solutions.
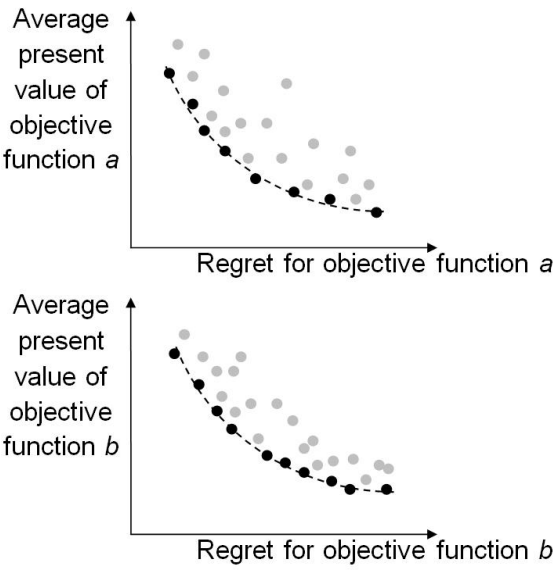

Figure 2 Expected trade-off between average objective function value and regret for each sustainability objective

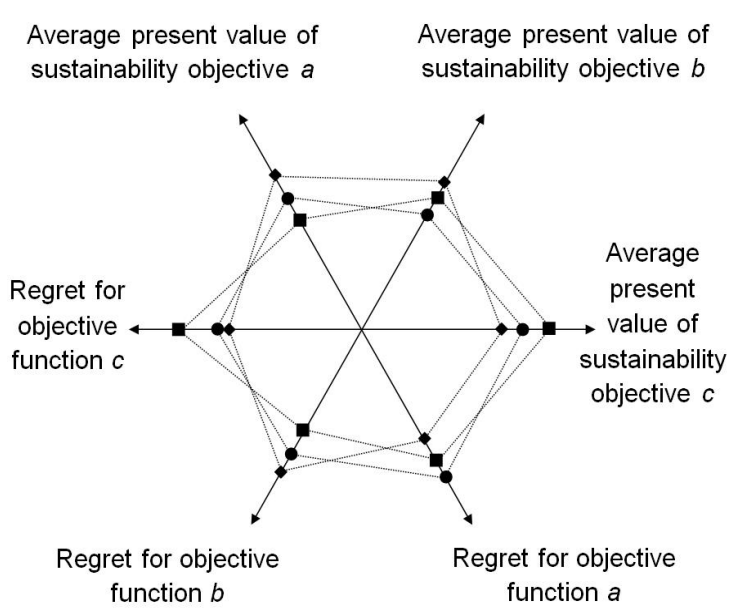

Figure 3 Expected trade-off between sustainability objectives for the average objective function value and regret

\section{PROPOSED FRAMEWORK FOR THE ROBUST OPTIMAL SEQUENCING OF URBAN WATER SUPPLY SOURCES}

In this section, the overall sequencing framework is introduced, which includes the two concepts introduced in the previous section. The proposed framework for the robust optimal sequencing of water supply sources consists of two stages. Firstly, the problem must be formulated correctly. This includes setting the planning horizon and staging interval; selecting sustainability objectives; and choosing possible water supply options. Secondly, the robust sequencing plan must be developed. This consists of the multi-objective optimization approach discussed in Section 2. The proposed framework is adaptive, allowing long term plans to be reviewed and re-optimised at regular intervals to take account of changes in circumstances and the availability of new data and information.

\subsection{Problem Formulation}

In the problem formulation stage, the sustainability objectives, planning horizon and possible water supply options need to be selected, as shown in Figure 4. 
Beh et al. Development of a modeling framework for optimal sequencing of water supply options...

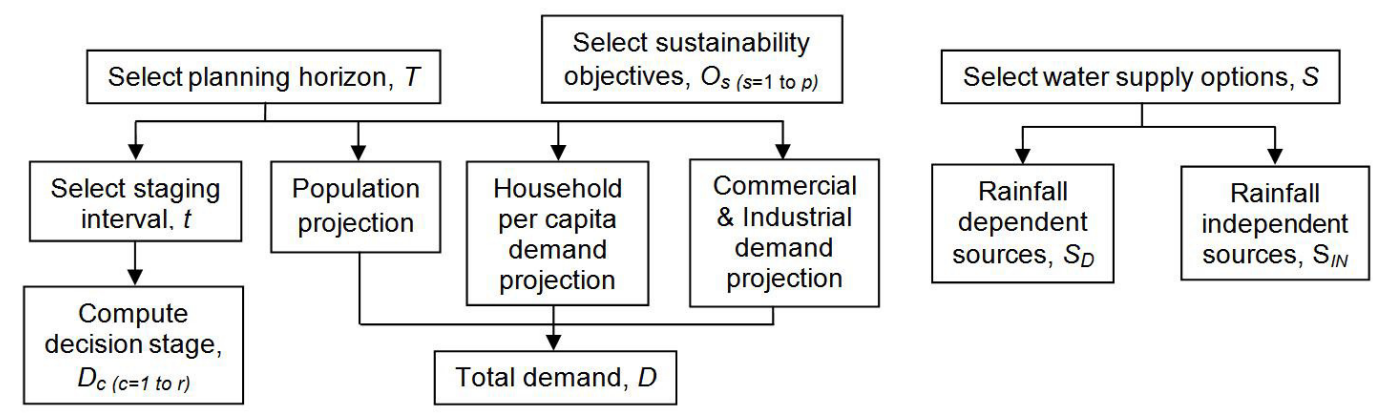

Figure 4 Problem formulation process

\section{Selection of Potential Water Supply Options}

Sequencing to safeguard regional water supply involves consideration of various possible water supply options, $S$. These are the means by which water is sourced for supply to consumers (see Figure 4) and include dams, desalination plants, recycled water and water tanks, all of which need to be incorporated into the sequencing process. Water supply options, $S$ are categorised into rainfall dependent and independent sources. Reservoirs, stormwater, groundwater and tanks are all dependent sources, $S_{D}$. Independent sources, $S_{I N}$ include desalination plants and wastewater reuse.

\section{Selection of Sustainability Objectives}

The next step in the problem formulation process is the selection of appropriate sustainability objective(s), $O_{S}$ to be optimised in the sequencing process (Figure 4). Previous studies have shown that economic factors have been considered to be the most influential criteria in water sources sequencing problems (Becker \& Yeh, 1974; Martin, 1988). However, in recent years, environmental and social factors are playing an increasingly important role in water resources planning (Hellström, 2000). Examples of sustainability objectives for the sequencing process, $O_{S}$ include minimising system cost, as well as the environmental and social impacts, while maximising system reliability. To plan for a long time period, the benefits and impacts resulting from planning will need to be quantified and discounted to present values in order to evaluate the impact on future conditions.

\section{Selection of Planning Horizon}

Choosing the sequencing planning horizon, $T$, which is the period of time over which the sequencing plan will operate (Figure 4), is a crucial part of the proposed sequencing approach. The staging interval, $t$, which is the length of time between decision points in the sequencing plan, also has to be selected. This interval needs to reflect a realistic period for the assessment of planning decisions and the design life of supply options.

After the planning horizon and staging interval have been defined, the number of decision stages, $D_{S}$ can be computed by dividing the planning horizon by the staging interval. For example, adopting 100 years of planning horizon, $T$ and 10 years staging interval, $t$ make up 10 decision stages, $D_{C}$ in the sequencing process. At each decision stage, decisions have to be made about which of the water supply options will be introduced or expanded, and the corresponding capacity accounted for. In addition, any constraints must be accounted for. For example, available supply must always exceed demand. Adjustments must be made, therefore, if this objective appears in jeopardy. These constraints are likely to be different at each of the stages because of changes in both demand and supply over time. At each staging interval, different water supply options are considered, examples of which are given in Figure 5. For each option, a set of capacities is considered (Figure 5). This means that within each staging interval, each supply option can take any discrete capacity value ranging from its current capacity to the maximum possible value. 
Beh et al. Development of a modeling framework for optimal sequencing of water supply options...

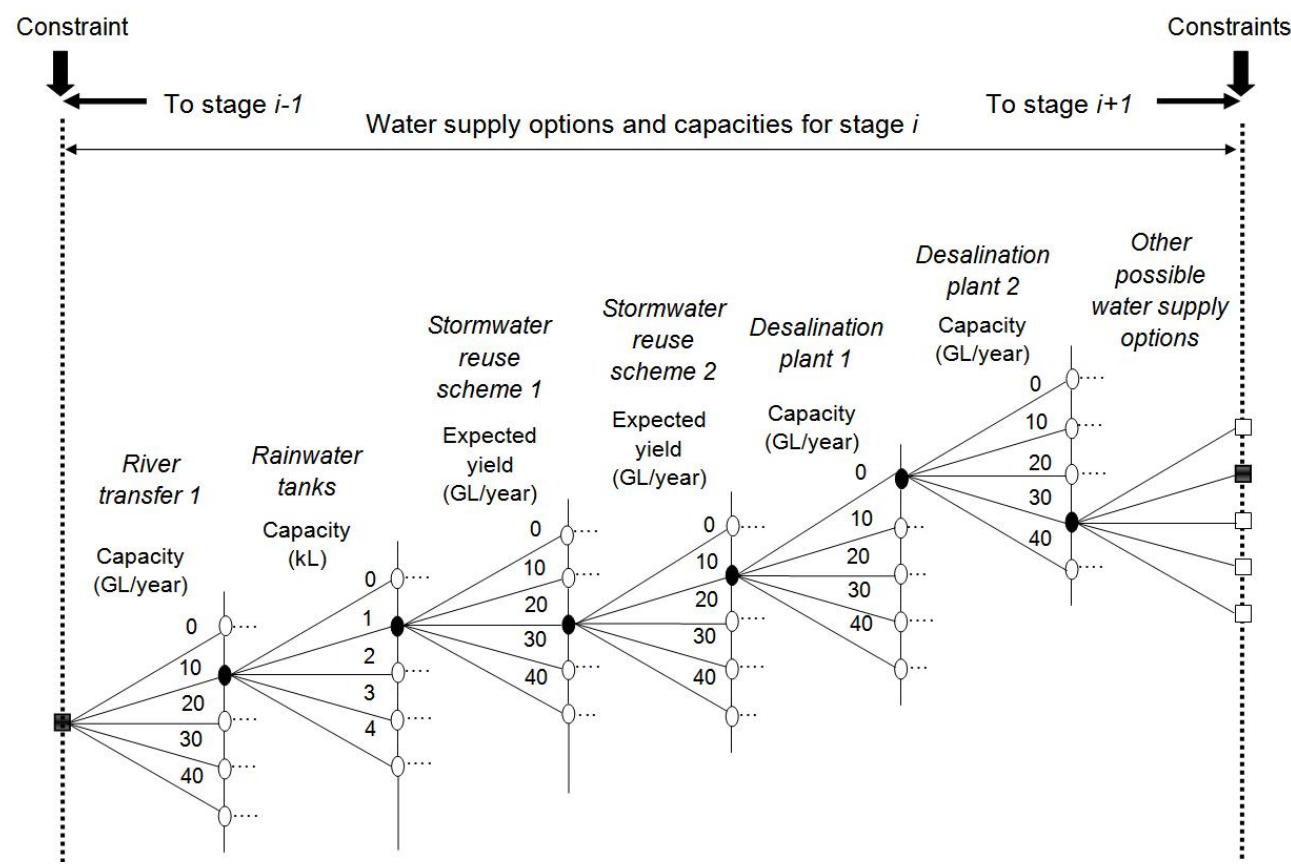

Figure 5 Flow diagram of one possible combination of water supply and capacity options selected at one decision point, $i$, in a water supply sequencing problem of $N$ stages

\subsection{Robust Sequencing Process}

The second stage of the proposed approach is the robust sequencing process, which consists of a multi-objective evolutionary optimization algorithm and a simulation model (e.g. WaterCress and REALM). It is necessary that the simulation and optimisation models are linked in order to assess whether the constraint that supply meets or exceeds demand at each decision point is met.

Figure 6 shows the process of robust sequencing. Firstly, an initial population of sequences, $\mathrm{P}_{G}$, is generated by randomly selecting values for each of the decision variables for each member of the population. Then, a number of scenarios representing uncertain future conditions (e.g. rainfall patterns, electricity prices and population growth), $\mathrm{U}_{C}$, are selected for each sequence to be assessed. After that, values of each of the sustainability objectives (e.g. cost and greenhouse gas emissions) has to be calculated for each of the uncertain scenarios for each of the sequences in the population (Figure 6).

Next, the feasibility of each sequence (i.e. whether supply meets or exceeds demand at each decision point) needs to be checked using the simulation model. Finally, the objective (fitness) functions that drive the searching process of the evolutionary optimization algorithm need to be calculated. As mentioned in Section 2, this includes the average and regret values for each of the sustainability objectives over the uncertain scenarios under consideration. In addition, whether a particular sequence is feasible or not also needs to be accounted for, as evolutionary algorithms are unable to deal with constraints explicitly. This can be done via the objective function (Figure 6) by using a penalty function or alternative mechanisms (Deb \& Gupta, 2006). 
Beh et al. Development of a modeling framework for optimal sequencing of water supply options...

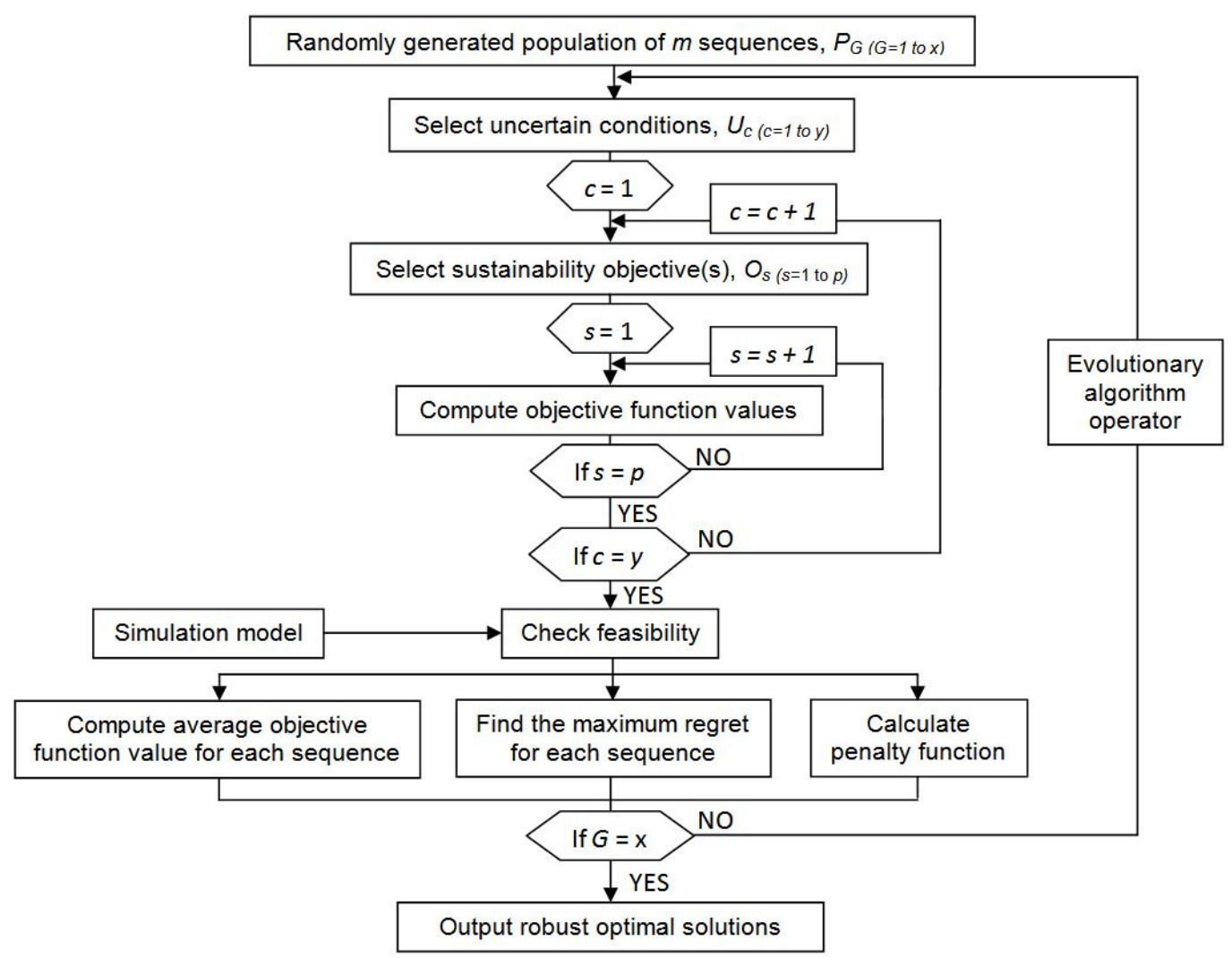

Figure 6 Robust sequencing process

Once the objective function values have been calculated, they are used to generate a new population of solutions (sequences) via the evolutionary operators of the selected algorithm. The above process is repeated until the selected convergence criteria have been met (e.g. pre-determined number of generations). The outputs can then be processed in a graphical fashion, as described in Section 2.

\section{SUMMARY}

The framework proposed in this paper incorporates uncertainty and sustainability into the sequencing of water supply projects at the regional scale. This includes adopting a long-term planning horizon because promoting sustainability of systems entails maximising the benefits of multiple sustainability objectives into the indeterminate future. The approach also utilises a multi-objective evolutionary optimisation algorithm in order to obtain optimal or near-optimal tradeoffs between competing sustainability objectives. Long term planning horizons promote increased uncertainties associated with the unforeseen future which requires a robust solution to best cope with the variety of potential conditions. The proposed approach incorporates robustness in the optimisation technique by including uncertain scenarios combined with robustness objectives to produce sequences which perform well across multiple situations. Finally, the proposed approach allows long term plans to be reviewed and re-optimised at regular intervals to take account of changes in circumstances.

The outcomes of the proposed approach will enable governments and water authorities to plan their water supply systems with greater confidence over a lengthy planning horizon. Complex systems of water supply will be more easily optimised, and water managers will have a clearer understanding of both financial and other costs, as well as benefits, given a range of possible future scenarios.

\section{ACKNOWLEDGEMENT}

The authors would like to thank Barbara Brougham for her editorial assistance with this paper. 
Beh et al. Development of a modeling framework for optimal sequencing of water supply options...

\section{REFERENCES}

Bankes S. (2010). Robustness, adaptivity, and resiliency analysis. AAAI Fall Symposium on Complex Adaptive System- Resilience, Robustness, and Evolvability, 2010.

Becker L. and Yeh W.WG. (1974). Optimal timing, sequencing and sizing of multiple reservoir Surface water supply facilities, Water Resources Research, 10(1), 57-62.

Connarty M. C. and G. C. Dandy (1996). Optimum Pricing and Capacity Expansion of Water Supply Headworks using Genetic Algorithms. Hydrology and Water Resources Symposium, 1996, 257-361.

Coombes P.J. and Lucas S. (2006). Towards sustainable water strategies in the Perth region of Western Australia: Inclusion of decentralized options. $1^{\text {st }}$ National HYDROPOLIS Conference, Perth, Western Australia, 2006.

Cui L. and Kuczera G. (2010). Coping with climate change uncertainty using robust multi-objective optimization: application to urban water supply systems, Practical Responses to Climate Change National Conference 2010, Melbourne, Australia.

Deb K., Pratap A., Agarwal S. and Meyarivan T. (2002). A fast and elitist multi-objective genetic algorithm: NSGA-II, IEEE Transactions on Evolutionary Computation, 6(2), 182-197.

Gethard K., Sae-Khow O. and Mitra S. (2011). Water desalination using carbon-nanotube-enhanced membrane distillation. ACS Applied Materials \& Interfaces, 3(2), 110.

Hellstrom D., Jeppsson U., Karrman E. (2000). A framework for systems analysis of sustainable urban water management, Environmental Impact Assessment Review, 20(3), 311-321.

Kundzewisz Z. and Somlyody L. (1997). Climatic change impact on water resources in a systems perspective, Water Resources Management, 11(6), 407-435.

Martin Q. (1987). Hierarchical algorithms for water supply expansion, Journal of Water Resources Planning and Management, 113(5), 677-395.

Mitchell C. (2007). Costing for sustainable outcomes in urban water systems: a guidebook, CRC for Water Quality and Treatment, Australia.

Paton F.L., Baulis J.P., Staniford B.S., Maier H.R. and Dandy G.C. (2009). Considering sustainability in the Planning and management of regional urban water supply systems: A case study of Adelaide's Southern system. $18^{\text {th }}$ World IMACS/MODSIM Congress, Cairns, Australia, July 2009, 3357-3363.

Tanaka, S.K., Zhu T., Lund J.R., Howitt R.E., Jenkins M.W., Pulido M.A., Tauber M., Ritzema R.S. and Ferreira I.C. (2006). Climate warming and water management adaption for California. Climate Change, 76(3-4), 361387. 